\title{
Premature rupture of the fetal membrane combined with subclinical chorioamnionitis negatively affects pregnancy outcomes by a mechanism associated with reduced levels of matrix metalloproteinase-2
}

\author{
HONGLI ZHANG ${ }^{1 *}$, LU WANG $^{2 *}$, JING WANG $^{1}$, JIANGRONG HEI $^{1}$ and CAILIAN RUAN ${ }^{2}$ \\ ${ }^{1}$ Department of Gynecology and Obstetrics, The Affiliated Hospital of Yan'an University; \\ ${ }^{2}$ School of Medicine, Yan'an University, Yan'an, Shaanxi 716000, P.R. China
}

Received June 18, 2014; Accepted January 13, 2015

DOI: $10.3892 /$ etm.2015.2559

\begin{abstract}
The present study aimed to investigate whether premature rupture of the fetal membrane, combined with subclinical chorioamnionitis, affects pregnancy outcomes. In addition, the association between premature rupture of the fetal membrane (PROM) and the levels of matrix metalloproteinase-2 (MMP-2), an inactive proenzyme that can be activated by other factors or signals in humans, was examined. In total, 80 pregnant patients with PROM were classified into the experimental and control groups, according to their final placental pathological diagnosis results. The 40 patients in the experimental group suffered from subclinical chorioamnionitis, while the 40 patients in the control group exhibited no lesions of the placenta or fetal membrane. Tissue samples were collected and the total protein and mRNA were extracted for western blot and quantitative polymerase chain reaction analyses. ELISA was performed in order to detect the levels of MMP-2 in the serum of the two groups of patients. The rates of cesarean section, puerperal infection, postpartum hemorrhage, preterm incidence, placenta accreta, residual placental blood and stillbirth were all significantly higher in the experimental group compared with the control group. In addition, the mRNA and protein expression levels of MMP-2 were reduced in the experimental group compared with the control group. ELISA results indicated that the serum MMP-2 concentrations were also reduced in the patients with PROM. Therefore, the present study demonstrated that the PROM, combined with subclinical chorioamnionitis, signifi-
\end{abstract}

Correspondence to: Dr Cailian Ruan, School of Medicine, Yan'an University, 38 Guanghua Road, Yan'an, Shaanxi 716000, P.R. China E-mail:w18868@126.com

*Contributed equally

Key words: subclinical chorioamnionitis, premature rupture of the fetal membrane, pregnancy outcomes cantly affected pregnancy outcomes and was associated with reduced levels of MMP-2.

\section{Introduction}

Chorioamnionitis is an important clinical factor that leads to premature rupture of the fetal membrane (PROM), and can be classified as subclinical or histological chorioamnionitis. During pregnancy, immune function is relatively low; thus, various pathogens from the vulva and cervix invade the uterus, which commonly results in subclinical chorioamnionitis (1). Subclinical chorioamnionitis may lead to inflammatory cell exudation, leukocyte infiltration edema, fibrous tissue proliferation and reduced elasticity/increased brittleness of fetal membrane, ultimately leading to premature rupture (2). Following the PROM, the environment of the uterus and vagina is altered in response, promoting bacterial proliferation and exacerbating the subclinical chorioamnionitis (3).

Chorioamnionitis is an easily overlooked condition, as the early clinical symptoms are not evident in the majority of pregnant patients and it is difficult to establish an accurate prenatal diagnosis (4). To date, there is no accurate method or index for predicting PROM in cases of subclinical chorioamnionitis, although there are a number of studies concerning the disease (5-9).

Matrix metalloproteinases (MMPs), including MMP-2, serve key functions in the development of various diseases by contributing to the degradation of type IV collagen, which is a major component of the extracellular matrix (10-12). MMP-2 is secreted as an inactive proenzyme and activated by other factors or signals. It has been reported that MMP-2 expression levels are reduced in maternal blood serum, and it is possible that MMP-2 concentrations are associated with preterm labor and fetal inflammatory responses (13). However, other studies demonstrated no association between MMP-2 concentration and preterm labor or fetal inflammatory responses $(14,15)$. Therefore, it is important to determine whether alterations in MMP-2 levels are associated with the PROM in patients with subclinical chorioamnionitis.

In the present study, the effects of PROM on pregnancy outcomes were investigated in cases of subclinical chorioam- 
nionitis. PROM, combined with subclinical chorioamnionitis, was observed to be associated with reduced levels of MMP-2.

\section{Materials and methods}

Patients. In total, 80 patients (age range, 24-32 years) that exhibited PROM were recruited and divided evenly into the control and experimental groups, according to their final placental pathology results. The 40 patients in the experimental group suffered from subclinical chorioamnionitis, while the 40 patients in the control group exhibited no lesions of the placenta or fetal membrane. All procedures were approved by the Ethics Committee of Yan'an University (Yan'an, China). Informed consent was obtained from all the patients or their families.

Patients included in the study agreed to undergo the following protocol: Newborns were immediately transferred into the intensive care unit for treatment after birth; the mother accepted ultrasound examination to monitor fetal and amniotic fluid conditions following admission to hospital; the mother underwent laboratory tests following admission to measure levels of C-reactive protein (CRP) and white blood cell count; and the neonatal outcomes were effectively followed-up. Patients were excluded if they exhibited any gestational diabetes, gestational hypertension, heart disease or if they refused to participate in the research or effective follow-up.

Parameters. Maternal clinical indicators, including age, gravidity, gestational age, body temperature, white blood cell count and CRP level were summarized and compared between the two groups. In addition, the rates of preterm incidence, cesarean section, puerperal infection, postpartum hemorrhage, placenta accreta, retained placenta and stillbirth were summarized and compared between the two groups.

Finally, the birth weights, Apgar scores, infection rates, incidence of respiratory distress syndrome, jaundice and neonatal mortality rates of the two groups of newborns were compared. The health conditions of the newborns were evaluated using Apgar scoring as described by Dai, Zuo and Li (16), which includes skin color, breathing, heart rate, reflection and muscle tension. The newborns with total scores $\geq 7$ were classed as healthy, those with scores $<7$ were considered to suffer from asphyxia.

Biopsy. Placenta samples were extracted from the center of the placental vertical section where the umbilical cord was attached during cesarean section. The size of the tissue samples was $\sim 1 \mathrm{x} 1 \mathrm{x} 1 \mathrm{~cm}$. Frozen $10-\mu \mathrm{m}$ sections were prepared according to normal procedures (17), followed by fixation with cold acetone.

Hematoxylin and eosin (HE) staining procedure. For conventional smear preparations, smear glass slides were fixed with $95 \%$ ethanol for $\geq 15 \mathrm{~min}$. The slides were then treated with phosphate-buffered saline (PBS) for $1 \mathrm{~min}$, hematoxylin for $10 \mathrm{~min}$, running water for $15 \mathrm{~min}$, eosin for $1 \mathrm{~min}, 95 \%$ ethanol for $1 \mathrm{~min}$ and $100 \%$ ethanol for $2 \mathrm{~min}$. Stained slides were cover-slipped with Permount. Finally, the HE-stained cells were examined under an SMT light microscope (1:7; binocular; Shanghai Milite Precise Instrument Co., Ltd.,
Shanghai, China) at x100-400 magnification. Milite Imaging Software was used for visualization (Shanghai Milite Precise Instrument Co., Ltd., Shanghai, China). Acetone was purchased from Sigma-Aldrich (St. Louis, MO, USA).

Enzyme-linked immunosorbent assay (ELISA). MMP-2 concentrations in the sera of the patients were determined using a commercially available ELISA kit (MMP-2 human ELISA kit; Life Technologies, Grand Island, NY, USA). The experiment was repeated independently at least six times. The results are expressed as the mean \pm standard deviation.

Reverse transcription-quantitative polymerase chain reaction (RT-qPCR) analysis. Total RNA was isolated using TRIzol reagent (Invitrogen Life Technologies, Carlsbad, CA USA) and mRNA was reverse transcribed to cDNA using Reverse Transcriptase M-MLV (Takara Biotechnology Co.,Ltd, Dalian, China). PCR reactions were conducted using a One-Step SYBR PrimeScript RT-PCR kit II (Takara Biotechnology Co., Ltd). Relative expression levels of MMP-2 mRNA were normalized against GAPDH. The primer sequences for MMP-2 and GAPDH detection were as follows: MMP-2, F 5'-AGGCTT AACTGATTAAGGCAC-3' and R 5'-GATGGCTACGAA TTCGATAGC-3'; GAPDH, F 5'-CATGCGCCTCACTAG TCAGCT-3' and R 5'-TACGCTGAGGATACAGGATAC-3'. The qPCR was performed using a 7500 Real-Time PCR system (Applied Biosystems Life Technologies, Foster City, CA, USA). Each experiment was repeated three times.

Western blot analysis. Total protein was prepared from the tissue samples scraped from the uterus. The total proteins were separated on 10\% SDS-PAGE gels (Beyotime Institute of Biotechnology, Shanghai, China) and transferred onto polyvinylidene fluoride membranes (EMD Millipore, Billerica, MA, USA). The membranes were incubated with monoclonal mouse primary antibodies against MMP-2 or GAPDH (1:200; Santa Cruz Biotechnology, Inc., Santa Cruz, CA, USA) overnight at $4^{\circ} \mathrm{C}$. The membranes were subsequently washed in PBS then incubated with secondary antibody conjugated to peroxidase (1:5,000; Santa Cruz Biotechnology, Inc.) for $1 \mathrm{~h}$. Subsequent to rinsing three times, the membranes were visualized using an ECL chemiluminescence kit (EMD Millipore). GAPDH was used for normalization. The relative intensity of the target bands was analyzed by Quantity One 1-D analysis software, version 4.6 (Bio-Rad Laboratories, Hercules, CA, USA). Each assay was independently repeated three times.

Statistical analysis. All data were analyzed using SPSS software, version 18.0 (SPSS, Inc., Chicago, IL, USA). Measurement data are presented as the mean \pm standard deviation and the $t$-test was used for comparison between groups. The count data are presented using percentages and the $\chi^{2}$ test was used for comparison between groups. $\mathrm{P}<0.05$ was considered to indicate a statistically significant difference.

\section{Results}

Subclinical chorioamnionitis histologically affects the placenta and fetal membrane. Histochemical analysis by optical microscopy was used to investigate the placental 
Table I. Clinical indicators of the two maternal groups.

\begin{tabular}{|c|c|c|c|c|c|c|}
\hline Group & $\begin{array}{c}\text { Age } \\
\text { (years) }\end{array}$ & Gravidity & $\begin{array}{c}\text { Body } \\
\text { temperature }\left({ }^{\circ} \mathrm{C}\right)\end{array}$ & $\begin{array}{l}\text { Gestation age } \\
\text { (weeks) }\end{array}$ & $\begin{array}{l}\text { White blood cell } \\
\text { count }\left(\times 10^{9} / 1\right)\end{array}$ & $\begin{array}{l}\text { CRP level } \\
(\mathrm{mg} / \mathrm{l})\end{array}$ \\
\hline${ }^{\mathrm{a}}$ Experimental $(\mathrm{n}=40)$ & $28.0 \pm 2.0$ & $3.5 \pm 1.0$ & $37.0 \pm 0.2$ & $32.0 \pm 1.0$ & $14.0 \pm 3.0$ & $12.9 \pm 5.5$ \\
\hline${ }^{\mathrm{a} C o n t r o l}(\mathrm{n}=40)$ & $27.0 \pm 2.5$ & $3.0 \pm 1.0$ & $37.1 \pm 0.2$ & $36.0 \pm 1.0$ & $9.0 \pm 1.0$ & $7.0 \pm 3.5$ \\
\hline t-test & 1.975 & 2.236 & 2.237 & 17.888 & 10.000 & 5.724 \\
\hline P-value & $>0.05$ & $>0.05$ & $>0.05$ & $<0.05$ & $<0.05$ & $<0.05$ \\
\hline
\end{tabular}

${ }^{a}$ Expressed as the mean \pm standard deviation. CRP, C-reactive protein.

Table II. Comparison of the clinical outcomes of the two maternal groups [n (\%)].

\begin{tabular}{lccccccc}
\hline Group & $\begin{array}{c}\text { Incidence } \\
\text { of preterm }\end{array}$ & $\begin{array}{c}\text { Cesarian } \\
\text { section }\end{array}$ & $\begin{array}{c}\text { Postpartum } \\
\text { hemorrhage }\end{array}$ & $\begin{array}{c}\text { Puerperal } \\
\text { infection }\end{array}$ & $\begin{array}{c}\text { Placenta } \\
\text { accreta }\end{array}$ & $\begin{array}{c}\text { Retained } \\
\text { placenta }\end{array}$ & $\begin{array}{c}\text { Stillbirth } \\
\text { Experimental }(\mathrm{n}=40)\end{array}$ \\
Control (n=40) & $25(62.5)$ & $30(75.0)$ & $15(37.5)$ & $18(45.0)$ & $8(20.0)$ & $9(22.5)$ & $4(10.0)$ \\
$\chi^{2}$ & $8(20.0)$ & $10(25.0)$ & $4(10.0)$ & $7(17.5)$ & $4(10.0)$ & $3(7.5)$ & $0(0)$ \\
P-value & 37.267 & 50.000 & 20.881 & 17.600 & 3.922 & 8.823 & 10.526 \\
& $<0.05$ & $<0.05$ & $<0.05$ & $<0.05$ & $<0.05$ & $<0.05$ & $<0.05$ \\
\hline
\end{tabular}

Table III. Comparison of the neonatal outcomes of the two groups of newborns [n (\%)].

\begin{tabular}{|c|c|c|c|c|c|c|}
\hline Groups & $\begin{array}{c}\text { Apgar } \\
\text { score }<7\end{array}$ & Infection & $\begin{array}{c}\text { Respiratory } \\
\text { distress syndrome }\end{array}$ & Jaundice & $\begin{array}{l}\text { Neonatal } \\
\text { mortality }\end{array}$ & $\begin{array}{c}\text { Birth } \\
\text { weight }(\mathrm{kg})\end{array}$ \\
\hline Experimental group $(n=36)$ & $18(50.0)$ & $8(22.2)$ & $14(38.9)$ & $7(19.4)$ & $4(10.0)$ & $1.70 \pm 0.35$ \\
\hline Control group $(n=40)$ & $5(12.5)$ & $2(5.0)$ & $5(12.5)$ & $3(7.5)$ & $0(0)$ & $2.23 \pm 0.45$ \\
\hline$\chi^{2}$ & 32.727 & 12.588 & 18.250 & 6.080 & 10.526 & 5.879 \\
\hline P-value & $<0.05$ & $<0.05$ & $<0.05$ & $<0.05$ & $<0.05$ & $<0.05$ \\
\hline
\end{tabular}

pathology of the patients. The experimental group consisted of 40 patients with subclinical chorioamnionitis (Fig. 1A and B). By contrast, no lesions of the placenta or fetal membrane were observed in the 40 subjects in the control group (Fig. 1C and D). The data indicated that subclinical chorioamnionitis impacted histologically on the placenta and fetal membrane.

PROM combined with subclinical chorioamnionitis significantly influences maternal clinical indicators, clinical outcomes and neonatal outcomes. Statistical analyses were performed in order to examine maternal clinical indicators, clinical outcomes and neonatal outcomes. No statistically significant difference was observed between the groups in age, gravidity or body temperature $(\mathrm{P}>0.05)$, while the gestational age, white blood cell count and CRP expression level did exhibit statistically significant differences $(\mathrm{P}<0.05$; Table I). The incidence of preterm birth and the rates of cesarean section, postpartum hemorrhage, puerperal infection, placenta accreta, retained placenta and stillbirth were all significantly higher in the experimental group compared with the control group $(\mathrm{P}<0.05$; Table II). With regard to the neonatal outcomes, 36/40 newborns survived in the experimental group, while 40/40 newborns survived in the control group. The average body weight of newborns in the experimental group was lower than that of the control group $(t=5.879 ; \mathrm{P}<0.05)$. Statistically significant differences were identified between the two groups, in the Apgar scores, infection rates, incidence of respiratory distress syndrome, incidence of jaundice and stillbirth rates of the newborns $(\mathrm{P}<0.05$; Table III). These results suggest that PROM combined with subclinical chorioamnionitis significant influenced maternal clinical indicators, clinical outcomes and neonatal outcomes.

MMP-2 concentrations in the serum of the experimental group are reduced, compared with those in the control group. Serum samples were collected and subjected to ELISA in order to investigate the effects of PROM combined with subclinical chorioamnionitis on MMP-2 serum concentrations. As presented in Fig. 2, the mean MMP-2 concentration in the experimental group was significantly reduced compared with that in the control group $(\mathrm{P}<0.05)$. These results suggest that reduced serum MMP-2 levels may be associated with rupture of the fetal membrane combined with subclinical chorioamnionitis. 

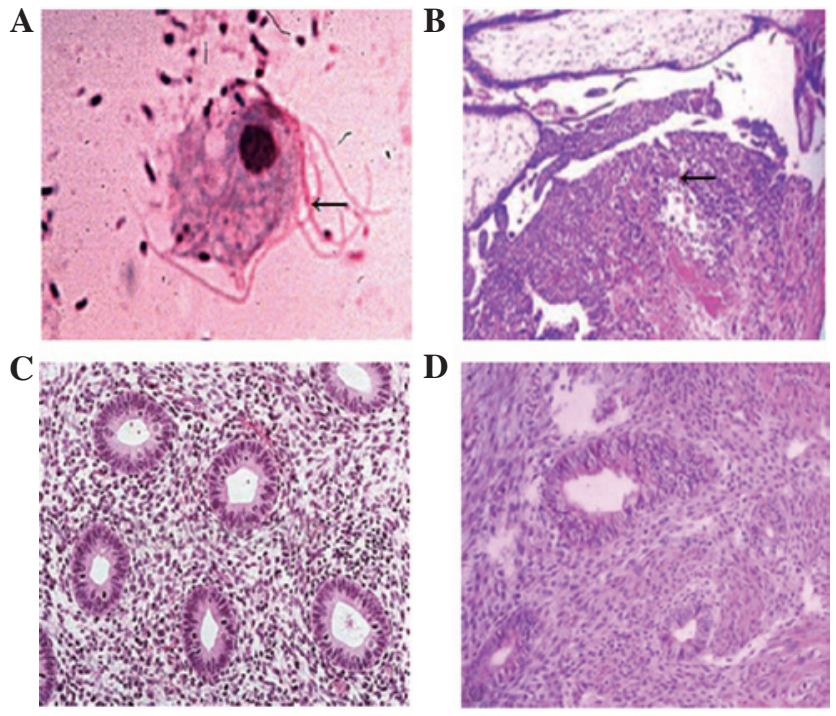

Figure 1. Representative microscopic images of pathological sections of (A) placental and (B) fetal subclinical chorioamnionitis (evident inflammatory cells marked with arrows), and (C) placental and (D) fetal normal endometrium tissues (magnification, $\mathrm{x} 100$ ).

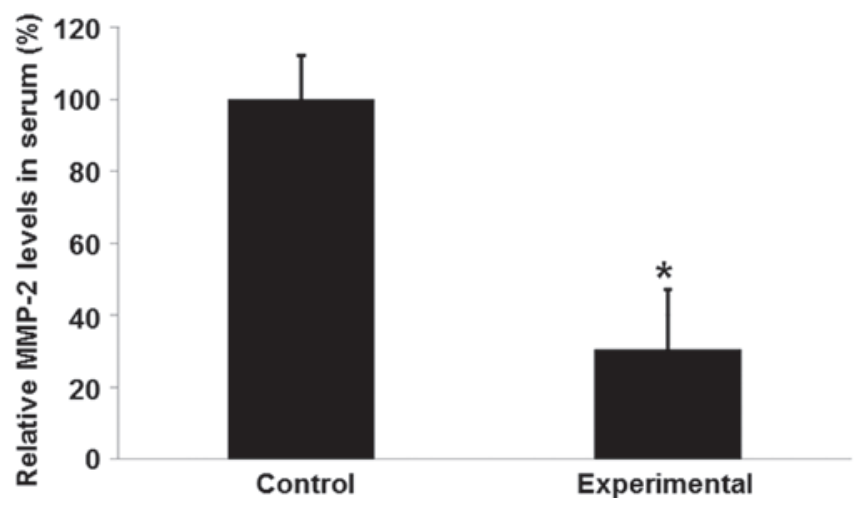

Figure 2. Levels of MMP-2 in the serum were detected by ELISA. MMP-2 levels in sera of the experimental group are reduced compared with those in the control group. Data are from six independent experiments and are expressed at the mean \pm standard deviation. ${ }^{*} \mathrm{P}<0.05$ vs. control. MMP-2, matrix metalloproteinase- 2 .

MMP-2 mRNA expression levels in the experimental group are reduced, compared with those in the control group. Total RNA was isolated from tissue samples from the patients in order to further determine if the MMP-2 levels are associated with rupture of the fetal membrane combined with subclinical chorioamnionitis. Analysis with qPCR indicated that the mRNA expression levels of MMP-2 in the experimental group were significantly lower than those in the control group (Fig. 3). The mean mRNA expression level of MMP-2 was $13.74 \%$ of the mean level in the control group (Fig. 3; $\mathrm{P}<0.05$ ). These results suggest that MMP- 2 mRNA expression levels are downregulated in patients with rupture of the fetal membrane combined with subclinical chorioamnionitis.

MMP-2 protein expression levels in the experimental group are reduced, compared with those in the control group. Total proteins were isolated from tissue samples from the patients in order to further determine if the MMP-2 protein expression levels may be associated with rupture of the fetal membrane

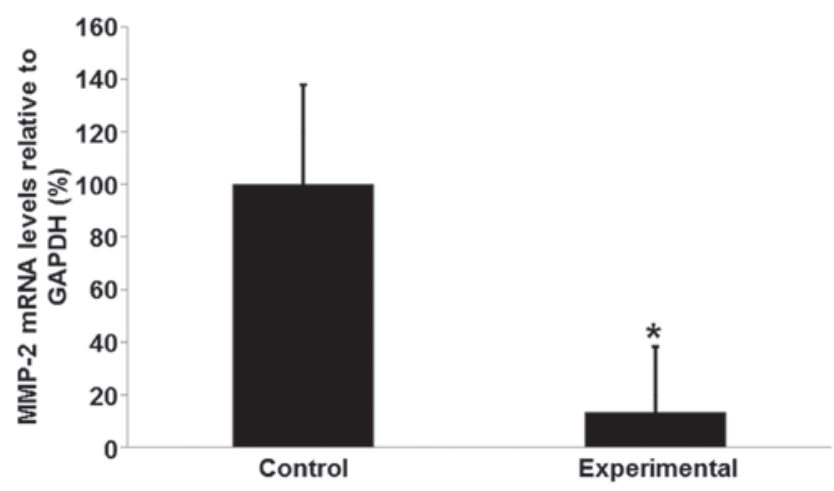

Figure 3. Total RNA was isolated and analyzed to measure MMP-2 mRNA expression levels by qPCR. Relative levels of MMP-2 mRNA were normalized against GAPDH mRNA levels. Each assay was repeated three times. ${ }^{*} \mathrm{P}<0.05$ vs. control. MMP-2, matrix metalloproteinase-2; qPCR, quantitative polymerase chain reaction.
A

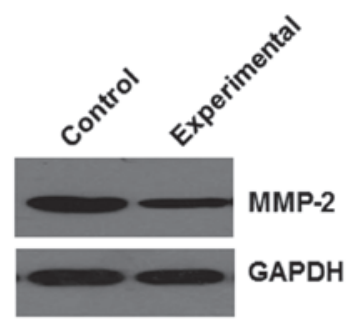

B

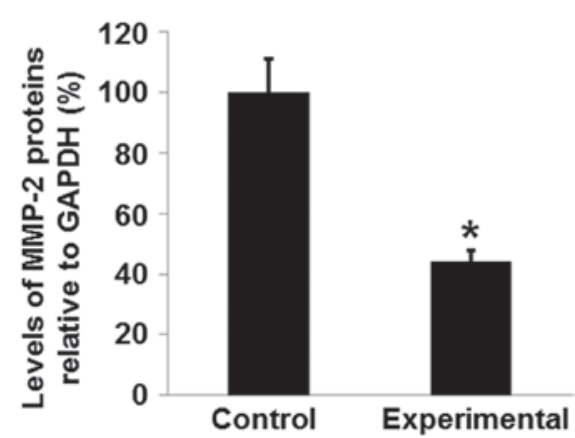

Figure 4. Western blot analysis. Tissue samples were collected from patients in the two groups. Total proteins were extracted for western blot analysis. (A) Representative blots (two repeat sets) and (B) relative intensities of the target MMP-2 bands, analyzed with Quantity One software. Each assay was repeated three times. ${ }^{*} \mathrm{P}<0.05$ vs. control. MMP-2, matrix metalloproteinase-2.

combined with subclinical chorioamnionitis. As presented in Fig. 4A, protein levels of MMP-2 in the experimental group were significantly reduced compared with those in the control group $(\mathrm{P}<0.05)$. The mean MMP-2 protein level in the experimental group was $42.23 \%$ of the level in the control group (Fig. 4B). These results suggest that MMP-2 is downregulated in the patients with rupture of the fetal membrane combined with subclinical chorioamnionitis.

\section{Discussion}

The clinical symptoms of subclinical chorioamnionitis may lead to long-term intrauterine infection that can increase the possibility of fetal distress or stillbirth, in addition to the rates of maternal placenta accreta, preterm birth and cesarean 
section (18). Furthermore, subclinical chorioamnionitis commonly leads to intrauterine adhesion, which affects the smooth discharge of maternal placenta following childbirth. As a result, the rates of retained placenta, postpartum hemorrhage and puerperal infection are higher in patients with PROM combined with subclinical chorioamnionitis (19). The present study demonstrated that the rates of preterm birth, cesarean section, puerperal infection, postpartum hemorrhage, placenta implantation, retained placenta and stillbirth were significantly higher in the experimental group than those in the control group. Although subclinical chorioamnionitis led to no evident clinical symptoms, it remained a threat to maternal health and safety.

Numerous studies have demonstrated that subclinical chorioamnionitis is closely associated with neonatal jaundice incidence, infection rate and respiratory distress syndrome incidence $(20,21)$. The maternal occurrence of subclinical chorioamnionitis has been demonstrated to enhance the risk of intrauterine infection. Furthermore, the hypoxic conditions of these fetuses were more severe than those of healthy fetuses; thus, the risk of asphyxia in childbirth was notably increased (22). In addition, subclinical chorioamnionitis has been demonstrated to reduce glucocorticoid-induced kinase expression in fetuses, affect fetal pulmonary gas exchange and induce pulmonary hypoplasia, resulting in poor clinical neonatal outcomes (23). In the present study, the Apgar scores and body weights of the experimental group of newborns were lower than those in the control group. Furthermore, the rates of neonatal mortality, respiratory distress syndrome, jaundice and infection were higher in the experimental group than in the control group. These results demonstrate that subclinical chorioamnionitis produces a negative impact on the clinical outcomes of newborns.

According to Been and Zimmermann (24), inflammatory responses in the body rapidly produce $\mathrm{CRP}$, a reactive protein located in the blood plasma, the concentration of which is positively correlated with the severity of inflammation and tissue injury. Therefore, the monitoring of maternal CRP expression levels has important clinical value in the prevention of chorioamnionitis. The present study demonstrated that the maternal CRP expression levels in the experimental group were significantly higher than in the control group. Therefore, dynamic monitoring of CRP expression levels in pregnant patients with PROM may aid in the choice of suitable treatments, and the improvement of the outcomes of pregnancy.

MMP-2 is important in various processes (25-27). A previous study indicated that MMP-2 may be associated with the PROM (21). However, other reports suggest that the relationship is uncertain $(14,15)$. The present study demonstrated that the MMP-2 mRNA and protein expression levels were reduced in the experimental group compared with the control group. In addition, ELISA results indicated that the serum MMP-2 concentrations were reduced in the patients with PROM. The limitation of the present study is the limited number of cases. In the future, we will increase the size of studied populations and investigate other proteins that are related to premature rupture of the fetal membrane combined with subclinical chorioamnionitis. In conclusion, PROM combined with subclinical chorioamnionitis was indicated to be associated with reduced levels of MMP-2.

\section{Acknowledgements}

The current study was supported by a grant from the Special Funds for the Construction of High-level Universities in Shaanxi Province, China (no. 2013SXTS02).

\section{References}

1. Vallejo MC, Kaul B, Adler LJ, et al: Chorioamnionitis, not epidural analgesia, is associated with maternal fever during labour. Can J Anaesth 48: 1122-1126, 2001.

2. Tang H: Correlation between the time of premature rupture of membranes and chorioamnionitis. Zhong Guo Bao Jian Ying Yang 20: 4247-4248, 2012 (In Chinese).

3. Liu J, Tang A and Chen M: Research on the correlation between the time of premature rupture of membranes and chorioamnionitis. Zhong Hua Zhong Xi Yi Xue Za Zhi 19: 14-15, 2012 (In Chinese).

4. Cui XF and Lou JY: The relationship between fetal fibronectin and chorionitis of premature rupture of membranes. Zhong Guo You Sheng Yu Yi Chuan Za Zhi She 6: 79-80, 2007 (In Chinese).

5. Aguin E, Van De Ven C, Cordoba M, Albayrak S and Bahado-Singh R: Cerclage retention versus removal following preterm premature rupture of membranes and association with amniotic fluid markers. Int J Gynaecol Obstet 125: 37-40, 2014.

6. Fortner KB, Grotegut CA, Ransom CE, et al: Bacteria localization and chorion thinning among preterm premature rupture of membranes. PLoS One 9: e83338, 2014.

7. Magee B and Smith G: Histological chorioamnionitis associated with preterm prelabour rupture of membranes at Kingston General Hospital: a practice audit. J Obstet Gynaecol Can 35: 1083-1089, 2013.

8. Chauhan SP and Ananth CV: Periviable births: epidemiology and obstetrical antecedents. Semin Perinatol 37: 382-388, 2013.

9. Stanek J and Biesiada J: Relation of placental diagnosis in stillbirth to fetal maceration and gestational age at delivery. J Perinat Med 42: 457-471, 2014.

10. Liotta LA, Tryggvason K, Garbisa A, Hart I, Foltz CM and Shafie S: Metastatic potential correlates with enzymatic degradation of basement membrane collagen. Nature 284: 67-68, 1980.

11. Stetler-Stevenson WG: The role of matrix metalloproteinases in tumor invasion, metastasis and angiogenesis. Surg Oncol Clin N Am 10: 383-392, 2001.

12. Stetler-Stevenson WG: Type IV collagenases in tumor invasion and metastasis. Cancer Metastasis Rev 9: 289-303, 1990.

13. Koucký M, Germanová A, Kalousová M, et al: Low maternal serum matrix metalloproteinase (MMP)-2 concentrations are associated with preterm labor and fetal inflammatory response. J Perinat Med 38:589-596, 2010.

14. Maymon E, Romero R, Pacora P, et al: A role for the $72 \mathrm{kDa}$ gelatinase (MMP-2) and its inhibitor (TIMP-2) in human parturition, premature rupture of membranes and intraamniotic infection. J Perinat Med 29:308-316, 2001.

15. La Sala GB, Ardizzoni A, Capodanno F, et al: Protein microarrays on midtrimester amniotic fluids: a novel approach for the diagnosis of early intrauterine inflammation related to preterm delivery. Int J Immunopathol Pharmacol 25: 1029-1040, 2012.

16. Dai B, Zuo F and Li Q: Correlation analysis of time of premature rupture of membranes with chorioamnionitis. Shong Guo Fu You Bao Jian 23: 3227-3228, 2008 (In Chinese).

17. Zhang W, Zhou L and Dang Y: Analysis of related factors of occult chorioamnionitis in mature pregnancy. Zhong Hua Yi Xue Za Zhi 9: 618-620, 2010 (In Chinese).

18. Chen X: Clinical analysis of 102 cases of premature rupture of membranes. Zhong Guo Yi Yao Zhi Nan 18: 193-200, 2012 (In Chinese).

19. He L: Clinical analysis of 117 cases of fetal distress caused by premature rupture of membranes. Zhong Guo Yi Yao Zhi Nan 30: 110-111, 2012 (In Chinese).

20. Bian X: Cause of premature rupture of membranes in early birth and progress in treatments. Zhong Wai Yi Liao 33: 191-192, 2012 (In Chinese).

21. Xie A, Di X, Chen X, et al: Factors influencing chorioamnionitis with premature rupture of membranes in early birth and the outcome of newborn. Zhong Hua Fu Chan Ke Za Zhi 47: 105-108, 2011 (In Chinese). 
22. Dang Y, Ma X and Zhang W: Analysis of pregnancy outcomes of 185 cases of premature rupture of membranes. Shan Xi Yi Xue Za Zhi 38: 69-77, 2009 (In Chinese)

23. Wang X, Li L and Cui S: The effect of type III collagen, CTGF and TNF- $\alpha$ in the pathologic mechanisms of premature rupture of membranes. Si Chuan Da Xue Due Bao 40: 58-60, 2009.

24. Been JV and Zimmermann LJ: Histological chorioamnionitis and respiratory outcome in preterm infants. Arch Dis Child Fetal Neonatal Ed 94: F218-F225, 2009.

25. Shi H, Liu L, Liu L, Geng J, Zhou Y and Chen L: $\beta$-Elemene inhibits the metastasis of B16F10 melanoma cells by downregulation of the expression of uPA, uPAR, MMP-2, and MMP-9. Melanoma Res 24: 99-107, 2014
26. Malz R, Weithaeuser A, Tschöpe C, Schultheiss HP and Rauch U: Inhibition of coagulation factor Xa improves myocardial function during CVB3-induced myocarditis. Cardiovasc Ther 32: 113-119, 2014.

27. Pandurangan A, Dharmalingam P, Sadagopan $S$ and Ganapasam S: Luteolin inhibits matrix metalloproteinase 9 and 2 in azoxymethane-induced colon carcinogenesis. Hum Exp Toxicol 33: 1176-1185, 2014. 